\title{
Is A1C Less Concordant with OGTT in Children as Compared in Adults? Data from a Hispanic Community in Arizona
}

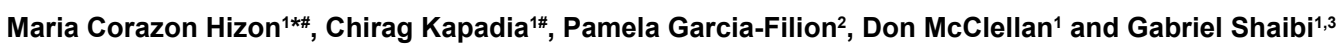

${ }^{1}$ Division of Endocrinology and Diabetes, Phoenix Children's Hospital, USA

${ }^{2}$ Trauma, Phoenix Children's Hospital, USA

${ }^{3}$ Center for Metabolic and Vascular Biology, Arizona State University, USA

\#Equal Contribution

\begin{abstract}
Early efforts to identify hyperglycemia and those at risk for developing type 2 diabetes (T2DM) are warranted. For decades, the diagnosis of T2DM has been based on plasma glucose (PG) criteria but recent recommendations include the use of $\mathrm{A} 1 \mathrm{C}$ for identifying hyperglycemia. These recommendations are based upon adult studies and data suggests that $\mathrm{A} 1 \mathrm{C}$ may be less concordant in children, as compared to adults. The purpose of our study was to compare A1C, fasting plasma glucose (FPG) and oral glucose tolerance test (OGTT) between adolescents and adults in Hispanic population at disproportionate risk for developing T2DM.
\end{abstract}

Methods: Data from self-identified as Latino, 91 overweight adolescents, and 406 overweight adults were assessed after an overnight fast for A1C, FPG and OGTT results. Receiver Operator Characteristics Curves for A1C vs. any hyperglycemia (prediabetes or diabetes) were then developed.

Result: $26(28.6 \%)$ of the adolescents and 209 (51.5\%) of adults exhibited hyperglycemia according to FPG and/OGTT. The prevalence of hyperglycemia as defined by an A1C $>5.7 \%$ was $30.8 \%$ in adolescents and $55.6 \%$ in adults. Of the 26 adolescents, hyperglycemic on FPG and /or OGTT only 9 had A1C $>5.7 \%$ for a sensitivity of $34.6 \%$. This in contrast to adults, where the sensitivity of $\mathrm{A} 1 \mathrm{C}>5.7 \%$ was $74.2 \%$. Positive predictive value (PPV) for the A1C threshold of $5.7 \%$ was $32.1 \%$ in adolescents vs. $73.7 \%$ in adults.

Conclusion: Concordance of A1C with other measures of hyperglycemia is lower in overweight Hispanic adolescents, as compared to overweight Hispanic adults.

Keywords: Type 2 diabetes; Metformin; Prediabetes; Hyperglycemic

Abbreviations: T2DM: Type 2 Diabetes Mellitus; FPG: Fasting Plasma Glucose; OGTT: Oral Glucose Tolerance Test; ADA: American Diabetes Association; A1C: Hemoglobin A1C; DPP: Diabetes Prevention Program; ROC: Receiver Operator Characteristic Curves; PPV: Positive Predictive Value; NPV: Negative Predictive Value; PG: Plasma Glucose

\section{Background}

The diagnosis of Type 2 Diabetes (T2DM) has been based on criteria for fasting plasma glucose (FPG) or 2 hour glucose on an oral glucose tolerance test (OGTT); more recent recommendations from the American Diabetes Association (ADA) include the use of hemoglobin A1C (A1C) for identifying hyperglycemia [1]. Recent data suggest that A1C may be less concordant with FPG and OGTT in children, as compared to adults [2,3]. We acknowledge that concordance studies do not answer larger questions about long-term risks associated with hyperglycemia. Nonetheless, the issue is relevant because many pediatric subspecialty centers have taken to treating 'prediabetes' with metformin in many cases in adolescents, on the basis of the Diabetes Prevention Program (DPP) results in adults [4]. Also, there is a more established practice of treating T2DM in children with metformin and insulin [5]. Data on concordance allows subspecialty centers to determine how to utilize $\mathrm{A} 1 \mathrm{C}$ in their treatment algorithms, if at all. The purpose of our study was to compare A1C, FPG, and OGTT between adolescents and adults in a Hispanic population at disproportionate risk for developing T2DM.

\section{Methods}

In participants self-identified as Latino, 91 overweight adolescents, and 406 overweight adults were assessed after an overnight fast for A1C, FPG, and OGTT results. All subjects were Latino in this population. Any subjects on medication for hyperglycemia were excluded, along with any subjects who identified themselves as having been previously diagnosed with diabetes or prediabetes. Other exclusions, such as hypertension, were not applied as the goal was to emulate a typical high volume clinic setting in which a variety of patients must be screened for diabetes. Inclusion criteria were Hispanic race, and overweight or obese status. Overweight or obese status for those age 10 to 20 was defined as BMI percentile $\geq 85^{\text {th }}$. For those $\geq 21$ years, overweight or obese status was defined as a BMI $>25 \mathrm{~kg} / \mathrm{m}^{2}$ [6].

Prediabetes was defined as FPG $\geq 100 \mathrm{mg} / \mathrm{dl}$, or 2 hour result on OGTT $\geq 140 \mathrm{mg} / \mathrm{dl}$; diabetes was defined as FPG $\geq 126$ or 2 hour result on OGTT $\geq 200$. Initial analysis at the A1C threshold of $5.7 \%$ was performed, to determine correlation between the Alc threshold of $5.7 \%$ and presence or absence of hyperglycemia on FPG or OGTT. Receiver Operator Characteristics curves (ROC) for A1C vs. any

*Corresponding author: Maria Corazon Hizon, Phoenix Children's Hospital, Division of Endocrinology and Diabetes, 1919 E Thomas Road, Phoenix, AZ 85016, USA, Tel: 602-933-0935; E-mail: pedsendo@phoenixchildrens.com

Received August 19, 2013; Accepted September 16, 2013; Published September 21,2013

Citation: Hizon MC, Kapadia C, Garcia-Filion P, McClellan D, Shaibi G (2013) Is A1C Less Concordant with OGTT in Children as Compared in Adults? Data from a Hispanic Community in Arizona. J Diabetes Metab 4: 290. doi:10.4172/21556156.1000290

Copyright: @ 2013 Hizon MC, et al. This is an open-access article distributed under the terms of the Creative Commons Attribution License, which permits unrestricted use, distribution, and reproduction in any medium, provided the original author and source are credited. 
hyperglycemia (prediabetes or diabetes) were then developed, to determine whether other Alc thresholds resulted in greater correlation with other measures of hyperglycemia.

\section{Results}

$26(28.6 \%)$ of the adolescents and 209 (51.5\%) of adults exhibited hyperglycemia according to FPG and/or OGTT. The prevalence of hyperglycemia as defined by an $\mathrm{A} 1 \mathrm{C} \geq 5.7 \%$ was $30.8 \%$ in adolescents and $55.6 \%$ in adults. However, despite similar percentages in the number of subjects defined as hyperglycemic, there was poor agreement between the tests for identifying hyperglycemic adolescents. Of the 26 adolescents hyperglycemic on FPG and/or OGTT only 9 had A1C $\geq$ $5.7 \%$, for a sensitivity of $34.6 \%$. This is in contrast to adults, where the sensitivity of $\mathrm{A} 1 \mathrm{C} \geq 5.7 \%$ was $74.2 \%$. Similarly, positive predictive value (PPV) for the A1C threshold of $5.7 \%$ was $32.1 \%$ in adolescents vs. $73.7 \%$ in adults.

A1C's performance marginally improved as a 'rule-out' test in adolescents, as of the 63 adolescents with an $\mathrm{AlC}<5.7 \%$, 46 were not hyperglycemic by FPG or $2 \mathrm{HrPG}$ (Specificity=70.8\%). Of the 181 adults with an $\mathrm{A} 1 \mathrm{C}<5.7 \%, 127$ did not exhibit elevated FPG or $2 \mathrm{HrPG}$ (Specificity $=64.5 \%)$. Similarly, negative predictive value (NPV) for the A1C threshold of $5.7 \%$ was $73.7 \%$ in adolescents and $70.2 \%$ in adults.

$5.7 \%$ is the recommended ADA threshold for prediabetes in adults, and the threshold at which most laboratories will report an 'abnormal' to the provider. Since correlation at this point was poor, we sought to define at what point correlation was better, by developing ROC curves for A1C vs. any hyperglycemia (prediabetes or diabetes).

As shown in the ROC curve and table (Figure 1 and Table 1), for overweight adolescents, using the presence of any hyperglycemia on FPG or OGTT as a de-facto gold standard, lower A1C values, such as $5.45 \%$, do raise sensitivity to more than $70 \%$, however, specificity then declines to near $50 \%$.

For overweight adults, the ROC curve and table (Figure 2 and Table 2) closely approximated the ADA recommendations, with values near $5.7 \%$ providing the best combination of sensitivity and specificity,

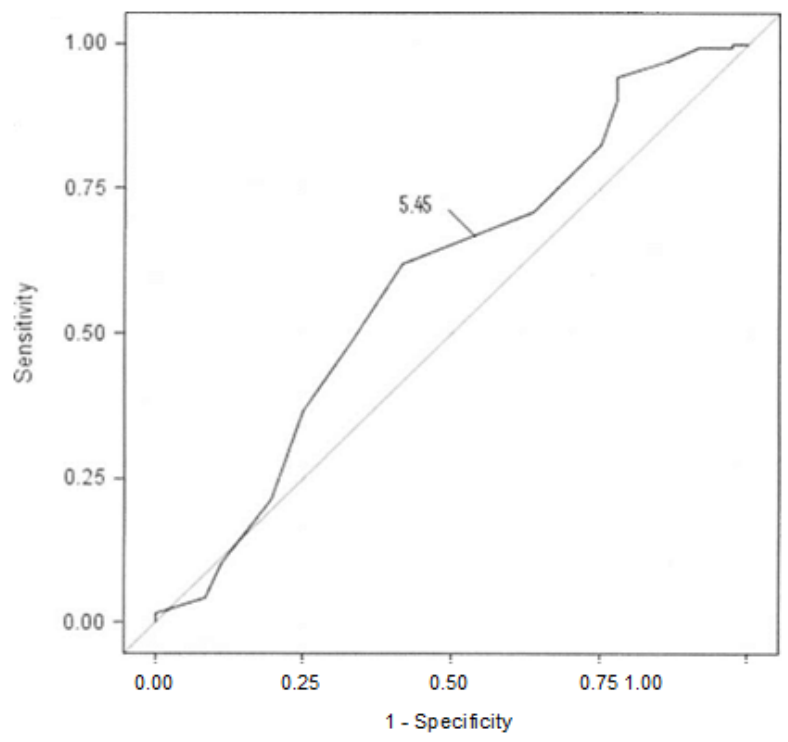

Figure 1: ROC Curve for Adolescents.

\begin{tabular}{|c|c|c|c|}
\hline Hemoglobin A1C & Sensitivity & Specificity & Sum of Sensitivity and Specificity \\
\hline 5.05 & 0.923 & 0.062 & 0.985 \\
\hline 5.15 & 0.885 & 0.138 & 1.023 \\
\hline 5.25 & 0.808 & 0.215 & 1.023 \\
\hline 5.35 & 0.769 & 0.338 & 1.107 \\
\hline 5.45 & 0.731 & 0.508 & 1.239 \\
\hline 5.55 & 0.615 & 0.585 & 1.2 \\
\hline 5.65 & 0.346 & 0.708 & 1.054 \\
\hline 5.75 & 0.231 & 0.815 & 1.046 \\
\hline 5.85 & 0.192 & 0.908 & 1.1 \\
\hline 5.95 & 0.192 & 0.938 & 1.13 \\
\hline 6.05 & 0.115 & 0.985 & 1.1 \\
\hline 6.15 & 0.038 & 1 & 1.038 \\
\hline
\end{tabular}

Table 1: A1C vs sensitivity, specificity, and sum in overweight and obese adolescents.

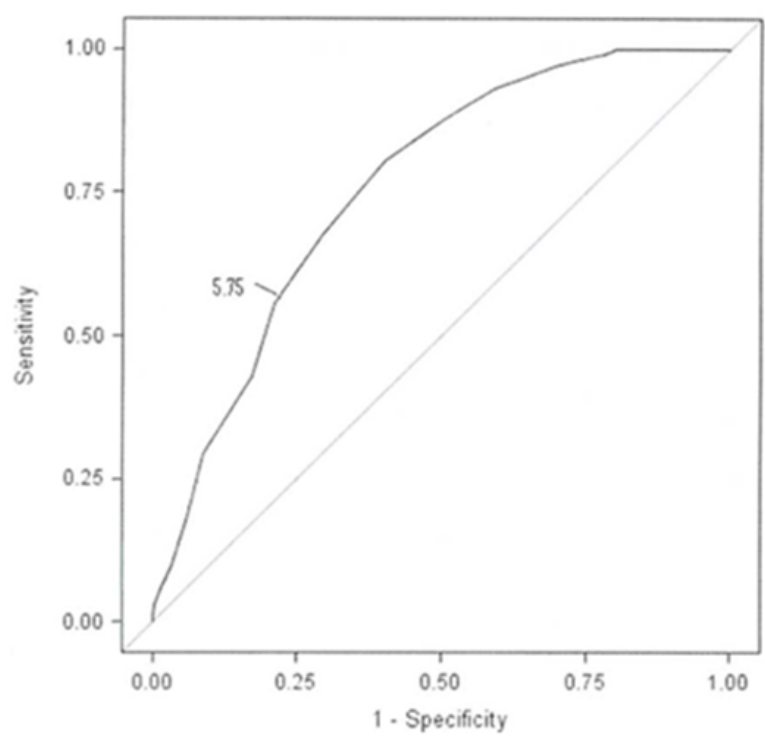

Figure 2: ROC Curve for Adults.

\begin{tabular}{|c|c|c|c|}
\hline Hemoglobin A1C & Sensitivity & Specificity & Sum of Sensitivity and Specificity \\
\hline 5.05 & 0.986 & 0.051 & 1.037 \\
\hline 5.15 & 0.971 & 0.091 & 1.062 \\
\hline 5.25 & 0.952 & 0.162 & 1.114 \\
\hline 5.35 & 0.923 & 0.259 & 1.182 \\
\hline 5.45 & 0.852 & 0.391 & 1.243 \\
\hline 5.55 & 0.823 & 0.528 & 1.351 \\
\hline 5.65 & 0.742 & 0.645 & 1.387 \\
\hline 5.75 & 0.627 & 0.782 & 1.409 \\
\hline 5.85 & 0.517 & 0.853 & 1.37 \\
\hline 5.95 & 0.426 & 0.914 & 1.34 \\
\hline 6.05 & 0.359 & 0.944 & 1.303 \\
\hline 6.15 & 0.316 & 0.964 & 1.28 \\
\hline 6.25 & 0.297 & 0.97 & 1.267 \\
\hline 6.35 & 0.244 & 0.985 & 1.229 \\
\hline 6.45 & 0.23 & 0.99 & 1.22 \\
\hline 6.55 & 0.206 & 1 & 1.206 \\
\hline
\end{tabular}

Table 2: A1Cvs sensitivity, specificity, and sum in overweight and obese adults.

when the presence of any hyperglycemia on FPG or OGTT is used as a de-facto gold standard. 
Citation: Hizon MC, Kapadia C, Garcia-Filion P, McClellan D, Shaibi G (2013) Is A1C Less Concordant with OGTT in Children as Compared in Adults? Data from a Hispanic Community in Arizona. J Diabetes Metab 4: 290. doi:10.4172/2155-6156.1000290

Page 3 of 3

Of note, in this study, there were no cases of diabetes in the obese adolescent population by FPG or OGTT, so all of the identified hyperglycemia was 'prediabetes'. There were 53 total cases of diabetes on FPG or OGTT in obese adults, and $\mathrm{AlC}$ was $\geq 5.7 \%$ in 51 of them.

\section{Conclusions}

In the overweight adult population, our correlation results closely approximate the recommendations put forth by the ADA.

In the overweight adolescent population, thresholds of $\mathrm{A} 1 \mathrm{C}$ to provide high concordance with FPG or OGTT are more difficult to identify.

Overall, we conclude that concordance of $\mathrm{AlC}$ with other measures of hyperglycemia is lower in overweight Hispanic adolescents, as compared to overweight Hispanic adults. Also, we conclude that for pediatric specialty clinics that have determined that clinical protocol is to treat 'pre-diabetes' as diagnosed on FPG or OGTT in adolescents with metformin, A1C does not offer additional guidance.

However, we do note that A1C is as good as FPG or OGTT, in adults, for identifying long-term complication risk, and that no similar studies regarding long-term complication risk have been carried out in adolescents or children; Thus, concordance studies provide an incomplete picture $[7,8]$. A1C may yet prove useful in primary care or urgent care settings, where the goal, rather than identification of prediabetes, is likely to be rule-out of significant hyperglycemia and diabetes requiring immediate treatment.

\section{Author's Contributions}

$\mathrm{MCH}, \mathrm{CK}$, and $\mathrm{GS}$ obtained and evaluated the research data. $\mathrm{MCH}$ and $\mathrm{CK}$ wrote the manuscript. PG helped in statistical analysis. GS, MCH, DM, CK, and PG contributed to discussion, reviewed/edited manuscript.

\section{Acknowledgement}

We are grateful to Joon Young Kim, PhD Student Kinesiology, Arizona State for his work in developing the ROC curves.

\section{References}

1. International Expert Committee (2009) International Expert Committee report on the role of the A1C assay in the diagnosis of diabetes. Diabetes Care 32: 1327-1334.

2. Lee JM, Wu EL, Tarini B, Herman WH, Yoon E (2011) Diagnosis of diabetes using hemoglobin $\mathrm{A} 1 \mathrm{c}$ : should recommendations in adults be extrapolated to adolescents? J Pediatr 158: 947-952.

3. Nowicka P, Santoro N, Liu H, Lartaud D, Shaw MM, et al. (2011) Utility of hemoglobin $A(1 c)$ for diagnosing prediabetes and diabetes in obese children and adolescents. Diabetes Care 34: 1306-1311.

4. Knowler WC, Barrett-Connor E, Fowler SE, Hamman RF, Lachin JM, et al (2002) Reduction in the incidence of Type 2 diabetes with lifestyle intervention or metformin. N Engl J Med 346: 393-403.

5. Copland KC, Silverstein J, Moore KR, Prazar GE, RaymerT, et al. (2013) Management of newly diagnosed diabetes mellitus in children and adolescents. Pediatrics 131: 364-382.

6. (1998) Clinical Guidelines on the Identification, Evaluation, and Treatment of Overweight and Obesity in Adults--The Evidence Report. National Institutes of Health. Obes Res 6: 51S-209S.

7. Kapadia C, Zeitler P; Drugs and Therapeutics Committee of the Pediatric Endocrine Society (2012) Hemoglobin A1c measurement for the diagnosis of Type 2 diabetes in children. Int J Pediatr Endocrinol 2012: 31

8. American Diabetes Association (2013) Standards of Medical Care in Diabetes--2013. Diabetes Care 36: S11-S66.
This article was originally published in a special issue, Diabetes: Case Studies handled by Editor(s). Dr. Vishwamitra Sharma, Nottingham University Hospital, UK 